\title{
The Breast Cancer Lifestyle Intervention Pilot Study
}

\author{
Elizabeth Kern1, Jennifer Chun1, Shira Schwartz1, Jessica Billig2, Erica Friedman², \\ Martha Eddy3, Deirdre Kiely1, Amber Guth', Deborah Axelrod1, Freya Schnabel1 \\ ${ }^{1}$ Department of Surgery, New York University Langone Medical Center, New York, USA \\ ${ }^{2}$ School of Medicine, New York University Langone Medical Center, New York, USA \\ ${ }^{3}$ Moving for Life, New York, USA \\ Email: Freya.Schnabel@nyumc.org
}

Received 24 July 2014; revised 20 August 2014; accepted 15 September 2014

Copyright (C) 2014 by authors and Scientific Research Publishing Inc.

This work is licensed under the Creative Commons Attribution International License (CC BY).

http://creativecommons.org/licenses/by/4.0/

(c) (i) Open Access

\section{Abstract}

Purpose: Weight loss after breast cancer diagnosis has been associated with a decrease in risk of breast cancer recurrence and mortality. The purpose of this study is to examine the barriers, acceptance, and sustainability of an exercise intervention program offered at our institution to overweight women with newly diagnosed breast cancer. Methods: The Breast Cancer Database was queried for women newly diagnosed with breast cancer and a body mass index (BMI) $\geq 25 \mathrm{~kg} / \mathrm{m}^{2}$. Eligible patients participated in the Moving for Life (MFL) exercise program for 16 sessions. Questionnaires were administered. Statistical analyses included descriptives and paired t-tests to summarize patient characteristics and assess changes over time. Results: Of 40 patients, 22 declined, 18 consented and $13(72 \%)$ completed the study. The mean age was 61 years (range: 38 76). The mean BMI was $31 \mathrm{~kg} / \mathrm{m}^{2}$. After the intervention, there was a decrease in weight and BMI $(p=0.04)$. The average weight loss was 10 lbs. Participants reported greater enjoyment of exercise $(p=0.02)$ and decreased pain related to treatment $(p=0.05)$. These initial positive results were not maintained after 6 months and 1 year. Conclusions: The MFL intervention had a high rate of acceptance among overweight women newly diagnosed with breast cancer. These results demonstrated significant benefits of exercise immediately after cancer diagnosis and highlight the importance of developing sustainable lifestyle interventions. Interventions targeted at modifiable lifestyle factors in women with early stage disease may provide benefit that is comparable to certain adjuvant systemic therapies. Therefore, adjuvant lifestyle interventions supported by clinicians may improve breast cancer survival outcomes.

\section{Keywords}

Breast Cancer, Lifestyle Intervention, Obesity

How to cite this paper: Kern, E., Chun, J., Schwartz, S., Billig, J., Friedman, E., Eddy, M., Kiely, D., Guth, A., Axelrod, D. and Schnabel, F. (2014) The Breast Cancer Lifestyle Intervention Pilot Study. Journal of Cancer Therapy, 5, 1031-1038. 


\section{Introduction}

Maintaining an ideal weight, defined as body mass index (BMI) $18.5-24.9 \mathrm{~kg} / \mathrm{m}^{2}$, after breast cancer diagnosis has been associated with improved survival outcomes, including decreased breast cancer recurrence and increased quality of life [1]-[7]. Physical activity is particularly important for obese women, who have an estimated 33\% increased risk of overall death and breast cancer-specific death, compared with non-obese women [8]. Interventions targeted at modifiable lifestyle factors in women with early stage disease may provide benefit that is comparable to certain adjuvant systemic therapies [9]. As a result, adjuvant lifestyle interventions may improve breast cancer survival outcomes in obese populations, and provide other health benefits as well.

Recent studies have shown that women who are physically active after breast cancer diagnosis have a $26 \%$ $40 \%$ lower risk of breast cancer recurrence, breast cancer death, and overall death, compared with sedentary individuals [6] [10] [11]. Although more studies are needed to confirm the findings, randomized control trials have suggested exercise may improve survival outcomes through beneficial changes in circulating levels of insulin, insulin-related pathways, inflammation, and possibly, immunity [11]-[13]. Additionally, exercise after diagnosis can lead to improvements in quality of life, fatigue, and body image [9].

In 2010, the American College of Sports Medicine established exercise guidelines for cancer survivors by reviewing the current evidence on safety and efficacy of exercise training during and after adjuvant cancer therapy. The committee determined that exercise programs specific for women diagnosed with breast cancer were safe during and after chemotherapy and radiotherapy [14]. With an increasing number of breast cancer survivors, groups at particularly high-risk of adverse survival outcomes, including overweight and obese women, are likely to achieve the greatest benefit from a lifestyle intervention, such as exercise.

There are many challenges to implementing weight loss interventions after cancer diagnosis. Weight loss programs are inherently difficult to implement and involve additional considerations in populations of cancer survivors. Side effects of cancer-related treatments, lack of social support, feelings of low self-efficacy, and other barriers can hinder the participation of newly-diagnosed cancer patients in weight loss programs [15]-[17]. Exercise programs are inherently difficult to maintain even in populations not facing additional challenges posed to women undergoing cancer treatment. The National Center for Health Statistics published a report in 2012 stating that only 21\% of all Americans engaged in adequate leisure-time aerobic and muscle-strengthening activities [18]. Cancer patients are more likely to exercise less as a result of adverse effects of the disease and treatment, such as treatment-related side effects, lack of social support and feelings of low self-efficacy [15] [16]. Among patients who participate in lifestyle interventions after diagnosis, poor adherence can limit the success of the intervention on improving survival outcomes, especially among overweight and obese individuals.

Few studies have examined challenges to implementing weight loss interventions, specifically in breast cancer populations [16] [19]. However, a prospective study of 196 breast cancer survivors in Taiwan examined the motivations for exercise frequency after a 6-month intervention. The results suggested that mental health, exercise barriers, and exercise outcome expectancy significantly contributed to differences in exercise frequency over this 6-month period. Additionally, baseline exercise frequency was the best predictor of overall exercise frequency during the study [19]. However, a major limitation of this study was the observational design, in which subjects were allowed to self-select their preferred exercise activity. Only regular exercise has been shown to yield long-term benefits for cancer survivors, but the motivation factors for maintaining healthy lifestyle changes after cancer diagnosis remain unclear. The purpose of this pilot study was to examine the barriers, acceptance, and sustainability of a lifestyle intervention among overweight women with newly diagnosed breast cancer.

\section{Materials and Methods}

\subsection{Study Population}

The Breast Cancer Database (BCD) at The New York University Langone Medical Center (NYULMC) is a prospective database that was established in January 2010 and includes all individuals undergoing newly diagnosed definitive breast cancer surgery. This database collects information regarding demographics, risk factors (personal and family history), treatment information, medical history, lifestyle factors and other information. Patients were eligible for this study if they enrolled in the BCD between December 2011 and July 2012 and had a $\mathrm{BMI} \geq 25 \mathrm{~kg} / \mathrm{m}^{2}$. All patients were within one year of definitive breast cancer diagnosis and surgical treatment. 
This study did not include women who could not exercise due to health related issues, who were pregnant, or who were $\geq 80$ years of age. This study was approved by the Institutional Review Board (IRB) of NYULMC.

\subsection{Study Design}

Eligible patients who consented to the study participated in a 90-minute exercise program that ran biweekly for eight weeks beginning in October 2012. This study incorporated the Moving for Life ${ }^{\mathrm{TM}} /$ Moving on Dance Exercise for Cancer Recovery ${ }^{\circ}$, a unique exercise program for breast cancer patients. Its goal is to introduce a safe and effective workout through dance exercise. What distinguishes the Moving for Life (MFL) program from other exercise programs is that it fosters group interaction and diverse personal expression, while also meeting fitness and health goals. This program has a 12-year track record at several New York hospitals and cancer centers for meeting breast cancer recovery and survivorship criteria as well as psychosocial parameters for being supportive and uplifting. The 90-minute exercise intervention sessions were led by the MFL founder and instructors formally trained by the MFL program. They were held in Moving Body Resources, an exercise studio located in New York City.

The Breast Cancer Database was queried for women newly diagnosed with breast cancer and a BMI $\geq 25$ $\mathrm{kg} / \mathrm{m}^{2}$. Eligible patients were consented and asked to fill out validated questionnaires [20]-[22] that employed a five-point Likert-type scale, with responses ranging from zero (not at all) to 4 (very much). These questionnaires were administered at the beginning of the MFL exercise program, at the end of the 16 sessions, and at 6-months and 1-year follow-up periods after program completion. Patients who chose not to participate in the exercise program intervention were asked to fill out a questionnaire to capture information on the barriers and reasons for declining study participation (Figure 1).

At the end of the study intervention, an educational cooking session was provided to participants by Cook for Your LIFE, a nonprofit organization that educates people affected by cancer in nutrition and cooking techniques to improve their health. Study participants were also given a copy of a nutritional cookbook, recipes for healthy eating, and a schedule of free MFL classes offered in the New York metropolitan area. Data were collected and analyzed between October 2012 and December 2013.

\subsection{Outcomes}

Primary outcomes measured in this study were rate of enrollment, acceptance rate, and changes in weight and

The Breast Cancer Lifestyle Intervention Pilot Study Timeline

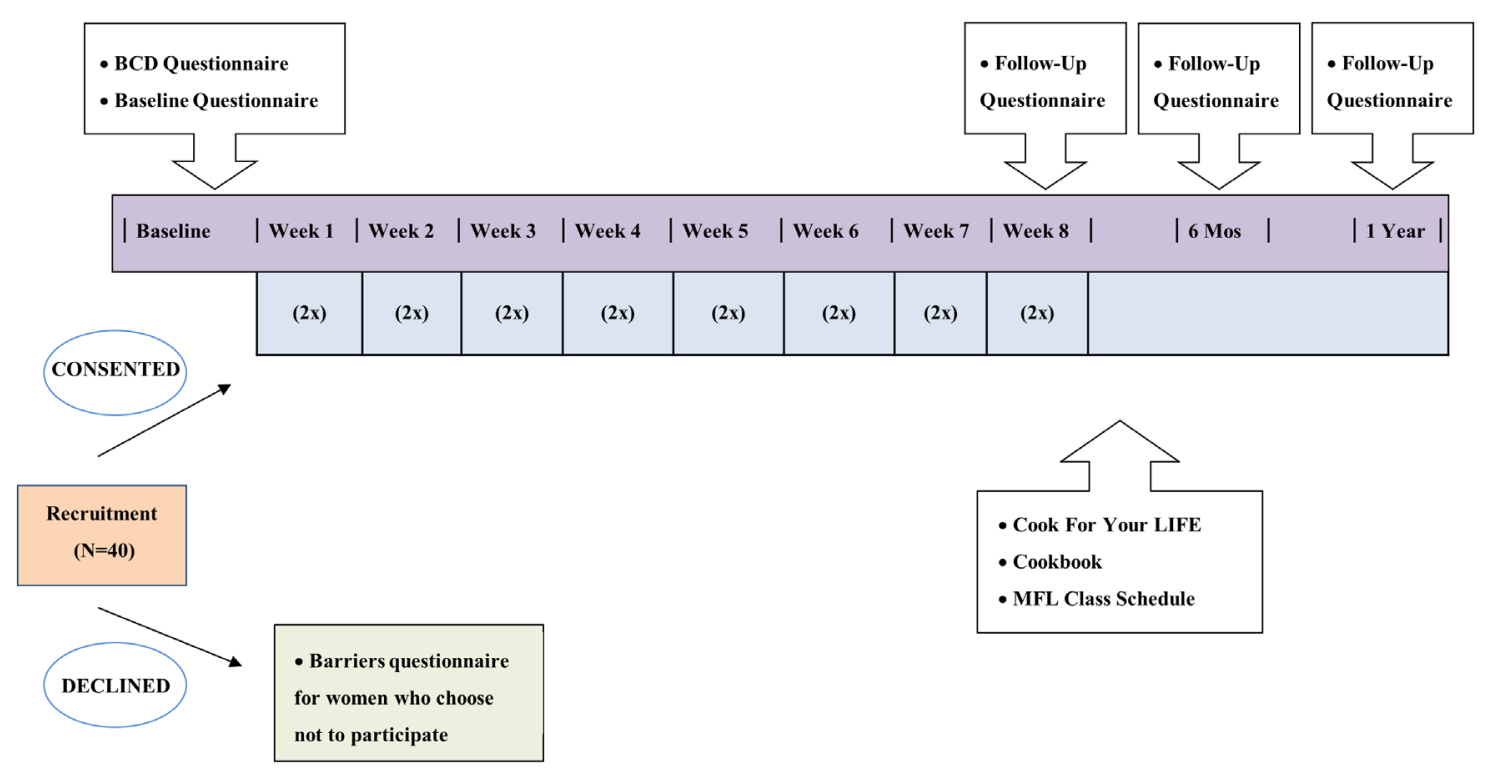

Figure 1. Study design. 
BMI among study participants. Secondary outcomes measured included changes in exercise enjoyment, strength, swelling, range of motion, stress, pain related to treatment, anxiety, depression, lack of exercise partners, and exercise barriers.

\subsection{Statistical Analyses}

Descriptive statistics were used to summarize patient characteristics and determine the rate of acceptance. Paired t-tests were used to determine if there were any significant differences in exercise attitudes and barriers to exercise sustainability before and after the intervention, and at the 6-month follow-up. Variables of interest included: age, weight, BMI, tumor characteristics, exercise enjoyment, strength, swelling, range of motion, stress, pain related to treatment, anxiety, depression, exercise partners and exercise barriers. All analyses were performed using SAS software (version 9.3, SAS Institute Inc.).

\section{Results and Discussion}

\subsection{Results}

During the study period, there were a total of 40 women who were eligible to participate in this study. A total of 22 (55\%) women declined to participate due to location, transportation limitations, and conflicts in schedule. 13 (72\%) out of the 18 women who consented to the MFL lifestyle intervention program completed the study. The mean age was 61 years (range: 38 - 76) and the mean baseline BMI was $31 \mathrm{~kg} / \mathrm{m}^{2}$ (range: 25 - 42). The majority of the women were Caucasian (72\%), had invasive ductal carcinoma (77\%), early stage breast cancer (67\%), and were estrogen receptor (ER) positive (67\%), progesterone receptor (PR) positive (56\%), and Her2-neu negative (83\%) (Table 1).

At the end of 8-weeks, there was a significant decrease in weight and BMI ( $p=0.04)$ (Table 2). The average weight loss was $10 \mathrm{lbs}$. Participants also reported a greater enjoyment of exercise $(p=0.02)$, as well as a decrease in pain related to treatment $(p=0.05)$ (Table 2). Positive changes were seen in other variables assessed as well. Increased strength, decreased swelling, decreased stress, and decreased depression were observed when overall averages were calculated, but these outcomes did not achieve statistical significance.

At the 6-month assessment period following the completion of the study, 10 out of the 13 participants completed follow-up questionnaires. There was an overall weight gain (average $3 \mathrm{lbs}$ ) and increase in BMI between the completion of the exercise program and the 6-month assessment period (Table 2). However, these results were not statistically significant. A decrease in strength and an increase in swelling, stress, pain related to treatment, anxiety, and depression were documented when comparing the overall averages of these results from the time of study completion to the 6-month follow-up assessment. These results were not statistically significant.

At the 1-year assessment period, 12 out of the 13 participants completed follow-up questionnaires. There was still a modest decrease in weight (average $2 \mathrm{lbs}$ ) and BMI between the completion of the exercise program and the 1-year follow-up period (Table 2). When the overall averages of these results were compared from the time of study completion to the annual follow-up, a decrease in strength, swelling, range of motion, pain related to treatment and a slight increase in depression were documented. However, these results did not achieve statistical significance.

At both 6 months and 1-year follow up assessments, the average reported scores describing exercise enjoyment and pain related to treatment remained the same when compared to the average scores recorded at the end of the MFL exercise program. When these variables were evaluated, the results were not statistically significant (Table 2).

\subsection{Discussion}

The overall goal of this study was to examine the barriers, acceptance and sustainability of an exercise intervention among overweight women newly diagnosed with breast cancer. In the short-term, the exercise intervention was successful in achieving moderate weight loss and overall positive health outcomes in the study population. While these positive results observed between baseline and intervention completion were not sustained over time, they provide important lessons for developing future exercise intervention initiatives. This study also raises the importance of developing programs that encourage sustainable lifestyle changes over time and improving health outcomes among breast cancer survivors in the near future. 
Table 1. Clinical characteristics.

\begin{tabular}{|c|c|c|}
\hline Variables & $N=18$ & $\%$ \\
\hline \multicolumn{3}{|l|}{ Race } \\
\hline Caucasian & 13 & 72 \\
\hline African-American & 3 & 16 \\
\hline Hispanic & 1 & 6 \\
\hline Mixed & 1 & 6 \\
\hline \multicolumn{3}{|l|}{ Histology } \\
\hline Ductal carcinoma in situ & 2 & 11 \\
\hline Invasive ductal carcinoma & 14 & 77 \\
\hline Invasive lobular carcinoma & 1 & 6 \\
\hline Invasive other & 1 & 6 \\
\hline \multicolumn{3}{|l|}{ Tumor stage } \\
\hline Stage 0 & 3 & 17 \\
\hline Stage I & 9 & 50 \\
\hline Stage II & 3 & 17 \\
\hline Stage III & 3 & 16 \\
\hline \multicolumn{3}{|l|}{ ER } \\
\hline Negative & 6 & 33 \\
\hline Positive & 12 & 67 \\
\hline \multicolumn{3}{|l|}{ PR } \\
\hline Negative & 8 & 44 \\
\hline Positive & 10 & 56 \\
\hline \multicolumn{3}{|l|}{ Her2-neu } \\
\hline Negative & 15 & 83 \\
\hline Positive & 3 & 17 \\
\hline
\end{tabular}

In this cohort of overweight breast cancer patients, there was a high rate of intervention acceptance and completion. Compliance during the study period can be attributed to a variety of factors. Most importantly, the MFL program provided a means of enjoyable exercise while also decreasing pain relating to disease treatment. It also provided psychological support to study participants by connecting them with exercise partners and other women going through similar experiences with regards to disease diagnosis and management. Additionally, program compliance led to self-reported feelings of improved well-being and empowerment among study participants. For these reasons, the lifestyle intervention was successful in positively impacting the health of study participants during the study intervention.

Overall, this exercise intervention successfully demonstrated a short-term positive health impact on a cohort of overweight women newly diagnosed with breast cancer. Immediately following the end of the MFL intervention period, the study demonstrated a statistically significant decrease in weight and BMI, with an average weight loss of $10 \mathrm{lbs}$. However, we were limited in our ability to quantify the impact of additional contributing factors to these results, such as changes in diet and psychological support obtained during the exercise intervention. The results of the study also revealed greater enjoyment of exercise and a decrease in treatment-related pain among study participants, which may impact long-term lifestyle changes. The exercise intervention was successful in achieving positive results during the 8-week study period, but these results were not sustainable over time. 
Table 2. Comparison of baseline, MFL program completion, 6-month follow-up, and 1-year follow-up assessment periods.

\begin{tabular}{|c|c|c|c|c|c|c|c|}
\hline Variables & $\begin{array}{l}\text { Baseline } \\
(\mathrm{N}=13)\end{array}$ & $\begin{array}{l}\text { MFL Program } \\
\text { Completion } \\
(\mathrm{N}=13)\end{array}$ & $p$-Value & $\begin{array}{l}\text { 6-Month } \\
\text { Follow-Up } \\
(\mathrm{N}=10)\end{array}$ & $p$-Value ${ }^{\mathrm{a}}$ & $\begin{array}{l}\text { 1-Year } \\
\text { Follow-Up } \\
(\mathrm{N}=12)\end{array}$ & $p$-Value ${ }^{\mathrm{b}}$ \\
\hline Weight [lbs (mean, range)] & $\begin{array}{c}190.3 \\
(145.0-253.0)\end{array}$ & $\begin{array}{c}185.4 \\
(134.0-235.0)\end{array}$ & $p=0.04$ & $\begin{array}{c}188.1 \\
(132.0-235.0)\end{array}$ & $p=0.59$ & $\begin{array}{c}183.3 \\
(132.0-239.0)\end{array}$ & $p=0.84$ \\
\hline BMI $\left[\mathrm{kg} / \mathrm{m}^{2}\right.$ (mean, range) $]$ & $31.8(24.8-43.8)$ & $30.9(22.7-40.0)$ & $p=0.04$ & $31.6(25.8-40.7)$ & $p=0.57$ & $30.8(24.5-40.3)$ & $p=0.80$ \\
\hline Enjoyment & $2(0-4)$ & $3(0-4)$ & $p=0.02$ & $3(1-4)$ & $p=0.78$ & $3(0-4)$ & $p=0.64$ \\
\hline Strength & $2(1-4)$ & $3(1-4)$ & $p=0.14$ & $2(0-3)$ & $p=0.07$ & $3(0-4)$ & $p=0.44$ \\
\hline Swelling & $2(0-4)$ & $1(0-3)$ & $p=0.06$ & $1(0-4)$ & $p=0.31$ & $1(0-2)$ & $p=0.39$ \\
\hline Range of Motion & $1(0-3)$ & $1(0-3)$ & $p=0.06$ & $1(0-4)$ & $p=0.69$ & $0(0-1)$ & $p=0.37$ \\
\hline Stress & $2(0-4)$ & $2(0-3)$ & $p=0.67$ & $2(0-4)$ & $p=0.83$ & $2(0-3)$ & $p=0.59$ \\
\hline Pain Related to Treatment & $1(0-4)$ & $1(0-3)$ & $p=0.05$ & $1(0-4)$ & $p=0.59$ & $1(0-2)$ & $p=0.75$ \\
\hline Anxiety & $1(0-3)$ & $1(0-3)$ & $p=0.75$ & $1(0-4)$ & $p=0.68$ & $1(0-3)$ & $p=0.59$ \\
\hline Depression & $0(0-2)$ & $1(0-1)$ & $p=0.67$ & $1(0-3)$ & $p=0.68$ & $1(0-2)$ & $p=0.50$ \\
\hline Lack of Exercise Partner(s) & $1(0-4)$ & $1(0-4)$ & $p=1.00$ & $2(0-4)$ & $p=1.00$ & $2(0-4)$ & $p=0.25$ \\
\hline
\end{tabular}

${ }^{\mathrm{a}}$ Comparison of 6-month follow-up to MFL program completion; ${ }^{\mathrm{b}}$ Comparison of 1-year follow-up to MFL program completion.

The 6-month and 1-year follow-ups assessed secondary endpoints, including exercise frequency and attitudes about exercise, and examined changes in and sustainability of behaviors and attitudes over time. In general, the study results demonstrated a modest weight loss, which was maintained throughout the year since the MFL program completion. However, individual results were highly variable and we cannot account for any weight loss due to a change in diet or psychological support obtained after the exercise intervention. Data collected 6months and 1-year following intervention completion indicated that significant positive changes were not sustainable over time for reasons including lack of exercise partners and inconvenient class location and schedule.

The desire to lose weight among study participants was sustained over time. In both the 6-month and 1-year follow-up questionnaires, study participants described current behaviors that included searching for available MFL classes and using smartphone applications and websites recommended during the intervention as weightloss aids. In addition, every study participant stated during follow-up that they would participate in an MFL exercise program if the program was offered at the NYULMC Cancer Center. However, this desire did not translate into a statistically significant weight loss. Nonetheless, follow-up assessment showed that encouragement given during the intervention to participants to sustain healthy exercise behaviors did have a lasting effect.

The study population did not continue exercising regularly with MFL programs offered in New York following study completion for reasons such as inconvenient class schedule and location, concurrent treatment side effects, and lack of exercise partners. These results demonstrate the need for an exercise program that is facilitated by a trained instructor and implemented regularly at a facility that is conveniently located and accessible to the patient population. In the future, our personal goal is to provide this exercise intervention program for breast cancer patients at the NYULMC Cancer Center.

It is important to note limitations to this study such as a small sample size, program location, scheduling conflicts, transportation, and Hurricane Sandy. Our ability to achieve statistically significant results at the 6-month and 1-year assessment periods was limited by the small sample size of study participants. The limitation of enrolling patients from one institution also contributed to the small study population, as many eligible patients could not participate in the exercise program due to location, transportation limitations, and conflicts in schedule. During the sixth week of the study intervention, New York City was severely affected by Hurricane Sandy. As a result, the average rate of attendance dropped from $80 \%$ to $50 \%$. Majority of the study participants reported power outages, lack of heat and water, and transportation difficulties. Hurricane Sandy and the impact it had on New York City and study participants affected program sustainability.

We acknowledge the importance of other lifestyle interventions, including diet, smoking, and alcohol con- 
sumption. However, for the purpose of this study, we focused on exercise, which has been shown to be the most effective intervention for weight loss and breast cancer risk reduction [5] [6]. In addition to physical activity, studies have shown that proper nutrition and a low-fat diet can contribute to weight loss and improved survival outcomes in breast cancer patients [9]. Although the study did not involve more extensive nutritional counseling, we tried to address this limitation by incorporating an educational cooking class and cookbook at the end of the exercise intervention. At the 6-month and 1-year follow-up, study participants reported increased awareness of dietary modifications and use of nutritional and dietary smartphone apps.

In the future, additional research should address some of the limitations of this study by evaluating the impact and sustainability of a more comprehensive lifestyle intervention program that incorporates both exercise and nutrition in a larger multi-institutional population.

\section{Conclusion}

This pilot study highlights the barriers, acceptance, and sustainability of a lifestyle intervention program offered by our institution, targeting exercise behaviors in overweight women who are newly diagnosed with breast cancer. The results from this study demonstrate the feasibility and short-term benefits of an exercise intervention supported by clinicians and the importance of developing sustainable lifestyle changes over time. The impact of lifestyle intervention programs has been proven to rival that of adjuvant therapies in early breast cancers without the adverse side effects. Additionally, lifestyle interventions offer unique opportunities for patient self-efficacy. Therefore, implementing an exercise program that is institutionally supported is a critical need in improving the health outcomes and survivorship of overweight women who are newly diagnosed with breast cancer. In order to achieve sustainable positive health outcomes, these exercise programs need to be enjoyable and accessible to all patients in a convenient location throughout the year.

\section{Acknowledgements}

This work was supported by the Charles Evans Foundation.

\section{Conflict of Interest Statement}

No financial disclosures or conflicts of interest were reported by the authors of this paper.

\section{References}

[1] Chlebowski, R.T., Aiello, E. and McTiernan, A. (2002) Weight Loss in Breast Cancer Patient Management. Journal of Clinical Oncology, 20, 1128-1143. http://dx.doi.org/10.1200/JCO.20.4.1128

[2] Kroenke, C.H., Chen, W.Y., Rosner, B. and Holmes, M.D. (2005) Weight, Weight Gain, and Survival after Breast Cancer Diagnosis. Journal of Clinical Oncology, 23, 1370-1378. http://dx.doi.org/10.1200/JCO.2005.01.079

[3] Nelson, N.J. (2012) Studies on How Lifestyle Factors May Affect Breast Cancer Risk and Recurrence. Journal of the National Cancer Institute, 104, 574-576. http://dx.doi.org/10.1093/jnci/djs213

[4] Obermair, A., Kurz, C., Hanzal, E., Bancher-Todesca, D., Thoma, M., Bodisch, A., et al. (1995) The Influence of Obesity on the Disease-Free Survival in Primary Breast Cancer. Anticancer Research, 15, 2265-2269.

[5] McTiernan, A., Kooperberg, C., White, E., Wilcox, S., Coates, R., Adams-Campbell, L.L., et al. (2003) Recreational Physical Activity and the Risk of Breast Cancer in Postmenopausal Women: The Women's Health Initiative Cohort Study. Journal of the American Medical Association, 290, 1331-1336. http://dx.doi.org/10.1001/jama.290.10.1331

[6] Friedenreich, C.M. (2001) Physical Activity and Cancer Prevention: From Observational to Intervention Research. Cancer Epidemiology Biomarkers and Prevention, 10, 287-301.

[7] (1998) Clinical Guidelines on the Identification, Evaluation, and Treatment of Overweight and Obesity in Adults—-The Evidence Report. National Institutes of Health. Obesity Research, 6, 51S-209S.

[8] Protani, M., Coory, M. and Martin, J.H. (2010) Effect of Obesity on Survival of Women with Breast Cancer: Systematic Review and Meta-Analysis. Breast Cancer Research and Treatment, 123, 627-635. http://dx.doi.org/10.1007/s10549-010-0990-0

[9] Ligibel, J.A. and Goodwin, P.J. (2012) New and Renew: Building the Case for Weight Loss in Breast Cancer. Journal of Clinical Oncology, 30, 2294-2296. http://dx.doi.org/10.1200/JCO.2012.42.5496

[10] Holmes, M.D., Chen, W.Y., Feskanich, D., Kroenke, C.H. and Colditz, G.A. (2005) Physical Activity and Survival af- 
ter Breast Cancer Diagnosis. Journal of the American Medical Association, 293, 2479-2486. http://dx.doi.org/10.1001/jama.293.20.2479

[11] Ballard-Barbash, R., Friedenreich, C.M., Courneya, K.S., Siddiqi, S.M., McTiernan, A. and Alfano, C.M. (2012) Physical Activity, Biomarkers, and Disease Outcomes in Cancer Survivors: A Systematic Review. Journal of the National Cancer Institute, 104, 815-840. http://dx.doi.org/10.1093/jnci/djs207

[12] Duggan, C., Irwin, M.L., Xiao, L., Henderson, K.D., Smith, A.W., Baumgartner, R.N., et al. (2011) Associations of Insulin Resistance and Adiponectin with Mortality in Women with Breast Cancer. Journal of Clinical Oncology, 29, $32-$ 39. http://dx.doi.org/10.1200/JCO.2009.26.4473

[13] Lawlor, D.A., Smith, G.D. and Ebrahim, S. (2004) Hyperinsulinaemia and Increased Risk of Breast Cancer: Findings from the British Women's Heart and Health Study. Cancer Causes and Control, 15, 267-275. http://dx.doi.org/10.1023/B:CACO.0000024225.14618.a8

[14] Schmitz, K.H., Courneya, K.S., Matthews, C., Demark-Wahnefried, W., Galvao, D.A., Pinto, B.M., et al. (2010) American College of Sports Medicine Roundtable on Exercise Guidelines for Cancer Survivors. Medicine and Science in Sports and Exercise, 42, 1409-1426. http://dx.doi.org/10.1249/MSS.0b013e3181e0c112

[15] Oldervoll, L.M., Kaasa, S., Hjermstad, M.J., Lund, J.Å. and Loge, J.H. (2004) Physical Exercise Results in the Improved Subjective Well-Being of a Few or Is Effective Rehabilitation for all Cancer Patients. European Journal of Cancer, 40, 951-962. http://dx.doi.org/10.1016/j.ejca.2003.12.005

[16] Mock, V., Frangakis, C., Davidson, N.E., Ropka, M.E., Pickett, M., Poniatowski, B., et al. (2005) Exercise Manages Fatigue during Breast Cancer Treatment: A Randomized Controlled Trial. Psycho-Oncology, 14, 464-477. http://dx.doi.org/10.1002/pon.863

[17] Milne, H.M., Wallman, K.E., Gordon, S. and Courneya, K.S. (2008) Impact of a Combined Resistance and Aerobic Exercise Program on Motivational Variables in Breast Cancer Survivors: A Randomized Controlled Trial. Annals of Behavioral Medicine, 36, 158-166. http://dx.doi.org/10.1007/s12160-008-9059-2

[18] Schiller, J.S., Lucas, J.W., Ward, B.W. and Peregoy, J.A. (2012) Summary Health Statistics for U.S. Adults: National Health Interview Survey, 2010. Vital and Health Statistics, 10, 1-207.

[19] Hsu, H.T., Dodd, M.J., Guo, S.E., Lee, K.A., Hwang, S.L. and Lai, Y.H. (2011) Predictors of Exercise Frequency in Breast Cancer Survivors in Taiwan. Journal of Clinical Nursing, 20, 1923-1935. http://dx.doi.org/10.1111/j.1365-2702.2010.03690.x

[20] Bandura, A. (1997) Self-Efficacy: The Exercise of Control. WH Freeman and Company, New York.

[21] Davis, A.H., Figueredo, A.J., Fahy, B.F. and Rawiworrakul, T. (2007) Reliability and Validity of the Exercise Self-Regulatory Efficacy Scale for individuals with chronic Obstructive Pulmonary Disease. Heart and Lung: The Journal of Acute and Critical Care, 36, 205-216. http://dx.doi.org/10.1016/j.hrtlng.2006.08.007

[22] Sechrist, K.R., Walker, S.N. and Pender, N.J. (1987) Development and Psychometric Evaluation of the Exercise Benefits/Barriers Scale. Research in Nursing and Health, 10, 357-365. http://dx.doi.org/10.1002/nur.4770100603 
Scientific Research Publishing (SCIRP) is one of the largest Open Access journal publishers. It is currently publishing more than 200 open access, online, peer-reviewed journals covering a wide range of academic disciplines. SCIRP serves the worldwide academic communities and contributes to the progress and application of science with its publication.

Other selected journals from SCIRP are listed as below. Submit your manuscript to us via either submit@scirp.org or Online Submission Portal.
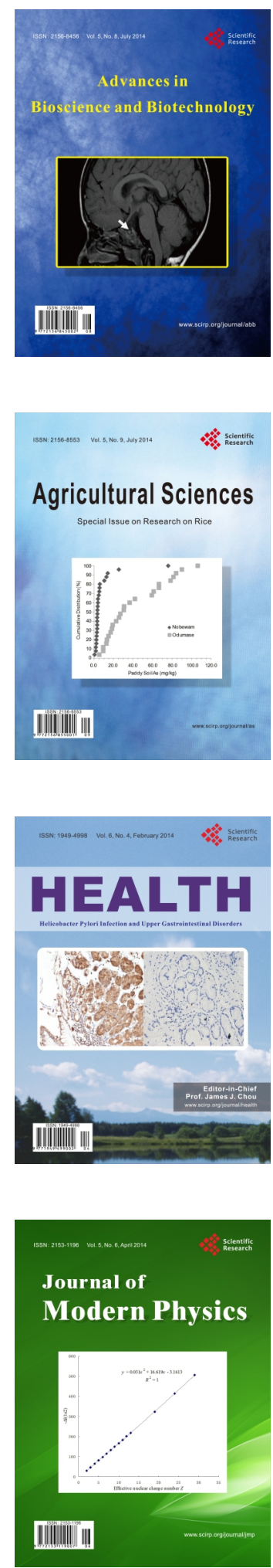
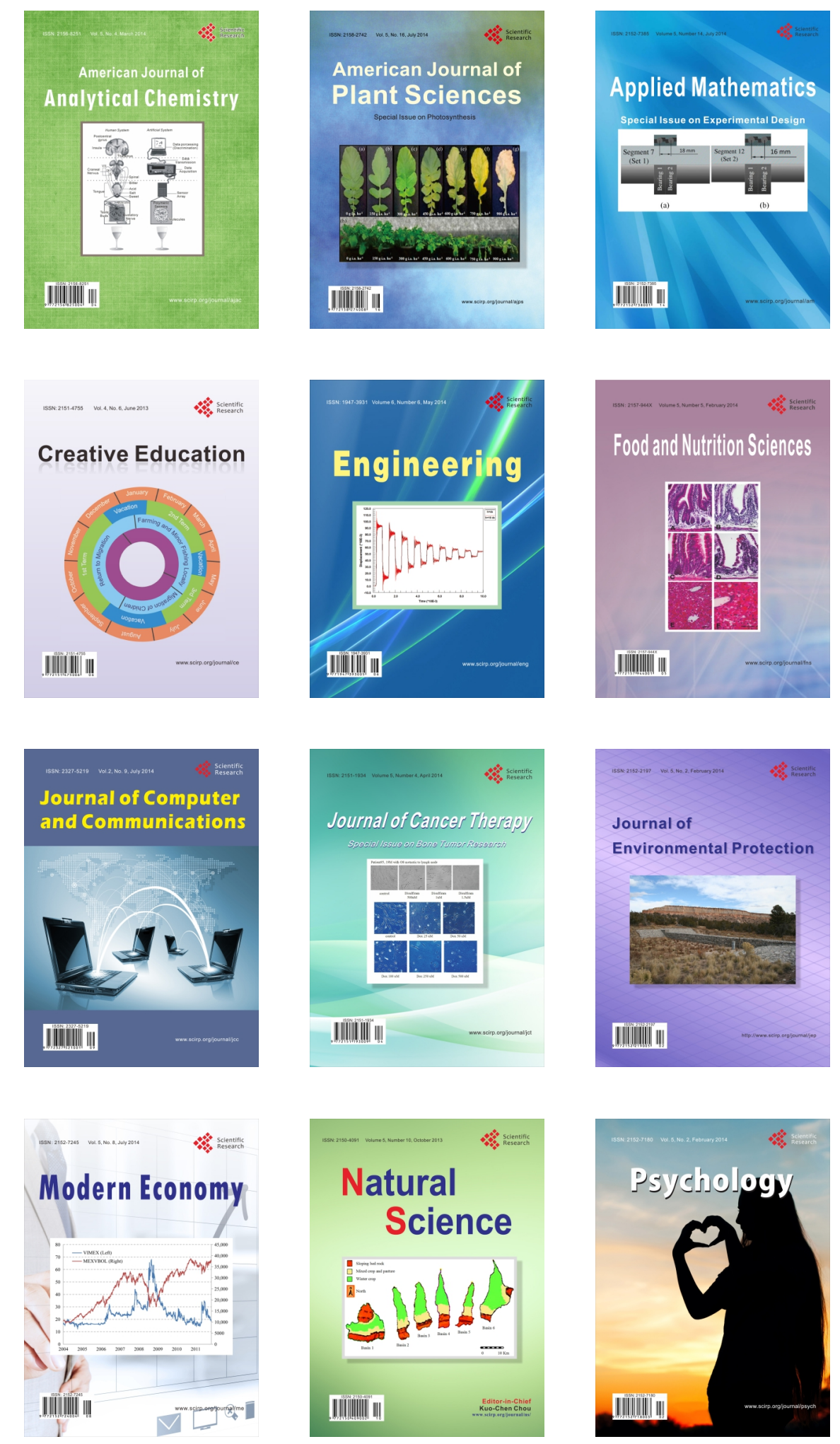